\title{
¿Hacia dónde se mueven los desempleados en Venezuela?'
}

\section{Where do the unemployed move in Venezuela??}

Recibido: 14 de marzo de 2016 - Revisado: 23 de mayo de 2016 - Aceptado: 24 de junio de 2016

Josefa Ramoni Perazzi ${ }^{2}$

Giampaolo Orlandoni Merli ${ }^{3}$

Surendra Prasad Sinha ${ }^{4}$

Elizabeth Torres Rivas ${ }^{5}$

Angel Zambrano ${ }^{6}$

\section{Resumen}

Según cifras oficiales en Venezuela tanto el desempleo como el PIB muestran una tendencia decreciente, lo cual lleva a preguntarse qué explica tal contradicción. La caída del desempleo en medio de un periodo de recesión económica solo es posible si los individuos emprenden actividades informales que los alejan del desempleo sin reportar su actividad, o si abandonan la búsqueda de empleo ante la imposibilidad de conseguir trabajo. A partir de la Encuesta de Hogares por Muestreo del 2012 y 2013, este estudio analiza la duración promedio del desempleo y el destino de los desempleados, utilizando para ello matrices markovianas de transición. Los resultados muestran una incidencia de desempleo de largo plazo superior al $40 \%$, lo que induce a algunos individuos a optar por cualquiera de estas dos alternativas.

\section{Palabras clave}

Desempleo, duración del desempleo, desempleo oculto, desempleo de largo plazo.

\begin{abstract}
According to official figures in Venezuela both unemployment and GDP show a decreasing trend, which leads one to wonder what explains this contradiction. The fall of unemployment in the midst of a period of economic recession is only possible if individuals undertake informal activities that distance them from unemployment without reporting their activity, or if they leave the job search in the face of the impossibility of finding work. Based on the Household Survey by Sampling for 2012 and 2013, this study analyzes the average duration of unemployment and the destination of the unemployed, using Markovian transition matrices. The results show an incidence of long-term unemployment of more than $40 \%$, which induces some individuals to opt for either of these two alternatives.
\end{abstract}

\section{Keywords}

Unemployment, duration of unemployment, hidden unemployment, long-term unemployment.
${ }^{1}$ El artículo es resultado de un proyecto de investigación que contó con el apoyo del Banco Central de Venezuela, la Universidad de Los Andes, Mérida, Venezuela y la Universidad de Santander, Bucaramanga, Colombia.

${ }^{2} \mathrm{PhD}$ y MSc en Economía de la University of South Florida, EEUU. MSc en Estadística de la Universidad de Los Andes, Venezuela. Economista de la misma universidad. Docente de la Universidad de Los Andes, Venezuela y de la Universidad de Santander, Colombia.

Correo electrónico: j.ramoni@udes. edu.co.

$3 \mathrm{PhD}$ en Estadística de la Universidad de Los Andes, Venezuela. MSc en Economía de Iowa State University, EEUU. Economista de la Universidad de Los Andes, Venezuela. Docente de la misma universidad y de la Universidad de Santander, Colombia.

Correo electrónico: gorlandoni@ udes.edu.co

${ }^{4} \mathrm{PhD}$ y MSc en Estadística Genética, Oregon State University, EEUU. Ingeniero Agrónomo de la Universidad de Bihar, India y Docente de la Universidad de Los Andes, Mérida, Venezuela.

Correo electrónico: sinha32@yahoo. com

5 Doctora en Formación, Empleo y Desarrollo de la Universidad de La Laguna, España. MSc en Estadística de la Universidad de Los Andes, Venezuela. Economista y docente de la misma universidad. Profesora titular de la Unicolombo, Colombia.

Correo electrónico: elisa@ula.ve

${ }^{6} \mathrm{MSc}$ en Estadística e Ingeniero de Sistemas de la Universidad de Los Andes de Venezuela. Profesor titular de la Universidad de Los Andes, Mérida, Venezuela.

Correo electrónico: angelz@ula.ve

Para citar este artículo use: Ramoni, J., Orlandoni, M., Prasad, M., Torres, E., \& Zambrano, A. (2017). ¿Hacia dónde se mueven los desempleados en Venezuela?. Civilizar Ciencias Sociales y Humanas, 17(32), 105-118. Doi: $10.22518 / 16578953.821$ 


\section{Introducción}

El desempleo es un problema que tiene repercusiones a varios niveles. A nivel nacional, el desempleo se traduce en capacidad productiva ociosa y desaprovechamiento de un recurso; marginamiento de una parte de la población, la cual no tiene acceso a la creación y disfrute de la riqueza. Pero, a diferencia de otros problemas macroeconómicos, como la inflación y la depreciación de la moneda, el desempleo es visto por los individuos como un problema tan personal y directo que algunos estarían dispuestos a canjearlo por inflación (Di Tella, MacCulloch, \& Oswald, 2001).

No todo desempleo puede ser tratado por igual. Según Ramoni (2012), se habla del desempleo friccional, como aquél resultante del rezago entre la continua entrada de individuos al mercado laboral y la asignación de éstos a los diferentes puestos de trabajo. Este se resuelve por sí solo a medida en que se conoce información acerca de los trabajadores/empleos disponibles; es de corta duración y le permite al individuo subsistir a partir de seguros de desempleo. A lo sumo, podrían introducirse ajustes para facilitar el proceso, divulgando la información.

El desempleo estructural, por otro lado, si amerita cuidado por cuanto, como su nombre lo indica, es la propia estructura económica la que lo genera, ya sea porque el mercado demanda trabajadores con capacidades diferentes a las que el sistema educativo produce; por el sesgo tecnológico que lleva a un desarrollo desigual entre sectores; por exceso de oferta laboral, propio de una economía deprimida, o el resultado de un largo periodo de recesión Así, se trata de una situación de más larga duración, que agota toda fuente de financiamiento posible y conlleva problemas de índole económico, familiar y social. Desde el punto de vista económico, se habla de ineficiente asignación de recursos, por cuanto los trabajadores terminan aceptando empleos por debajo de sus capacidades; desestimulo a la capacitación y pérdida de ingresos tanto durante el periodo de desempleo como a futuro, por obsolescencia del trabajador. Además, a mayor duración del desempleo, mayor probabilidad de abandono de la búsqueda. Desde el punto de vista familiar, el desempleo se asocia con pérdida de confianza del trabajador en sí mismo, menor esperanza de vida, menor nivel de formación académica de los hijos de desempleados y menores ingresos a futuro. Desde el punto de vista social, el desempleo de largo plazo margina a la porción de trabajadores que no logran posicionarse en el mercado, induciéndolos a la informalidad o, peor aún, generando delincuencia, prostitución y conflictos sociales.

Este estudio revisa el tiempo que en promedio demoran los individuos en abandonar la condición de desempleo y hacia qué estado se mueven cuando ello ocurre. Se recurre a matrices de transición markovianas aplicadas a datos de individuos entre 15 y 60 años de edad para el periodo 2012-2013 proporcionados por la Encuesta de Hogares por Muestreo (EHM) del Instituto Nacional de Estadística (INE) de Venezuela ${ }^{1}$. Los resultados indican que el tiempo promedio de búsqueda de empleo ronda los 11 meses, mayor para las mujeres y creciente con el nivel educativo. Dos tercios de los desempleados logran conseguir empleo, 40 $\%$ de ellos en el sector informal. Los demás, se dispersan en situaciones propias de la población económicamente inactiva.

El estudio se organiza en seis secciones. Después de esta introducción, la segunda sección plantea el problema a analizar, mientras que la tercera revisa el estado del arte de estudios en la materia. La cuarta sección resume la metodología de estudio, incluyendo la fuente de información. Las dos últimas secciones, muestran los resultados obtenidos y las principales conclusiones alcanzadas.

\section{Planteamiento del problema}

Según reporta el INE, el desempleo en Venezuela ha mostrado una marcada tendencia 
a disminuir desde que en los años 2002 y 2003 mostrase sus valores más altos $(16,2 \%$ en 2002 II y $19,2 \%$ en $2003-I)$. Extrañamente, esta aparente reducción del desempleo viene acompañada de una apreciable desaceleración de la economía, según el Banco Central de Venezuela (BCV). En efecto, a raíz del paro petrolero y el despido masivo de trabajadores de la estatal Petróleos de
Venezuela, la actividad económica se contrajo a una tasa promedio del $8,4 \%$ semestral durante los años 2002 y 2003 . A esta caída del producto interno bruto (PIB) le siguió un repunte de la actividad económica y posterior desaceleración sostenida, alcanzando valores incluso negativos, en medio del mayor y más largo auge de los precios del petróleo que la historia registra (ver gráfica 1).

Gr” ÿca 1

Tasa de crecimiento y tasa de desempleo en Venezuela (\%)

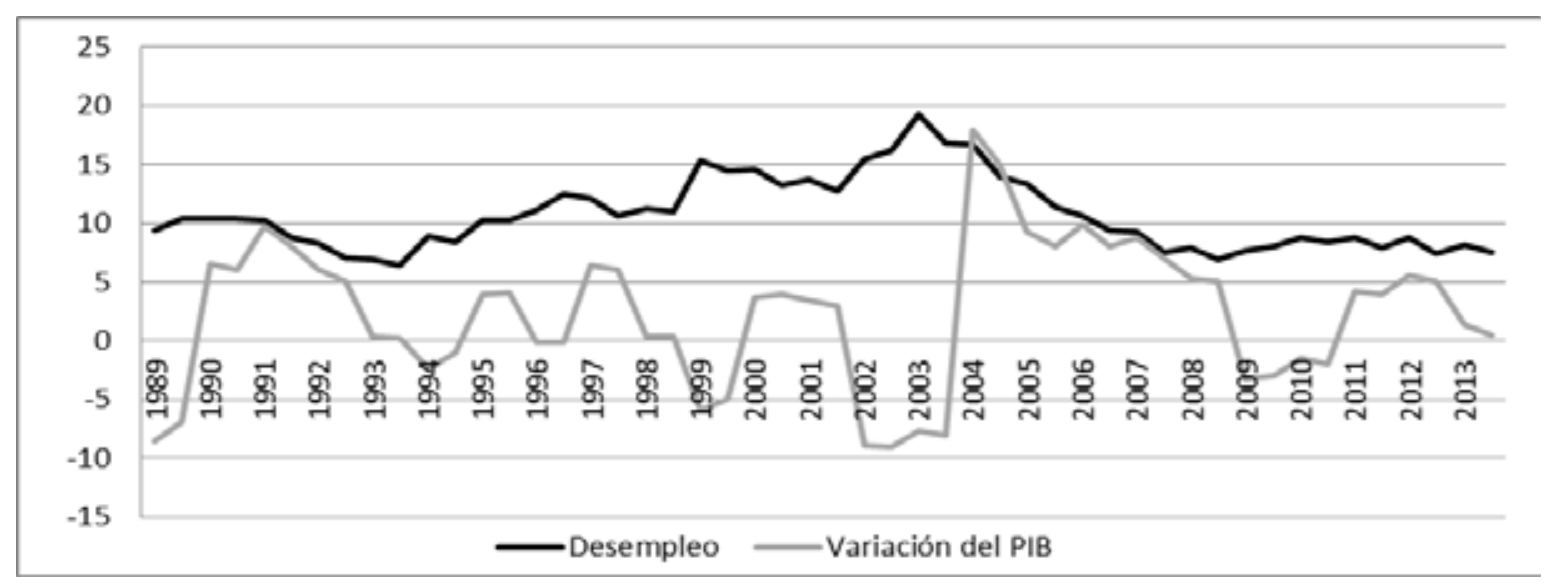

Fuente: elaboración propia con datos del INE y BCV, 1990-2014.

Tal como la teoría económica y la lógica sugieren, la solución al problema del desempleo pasa por la generación de producto, por lo que una caída simultánea de las tasas de desempleo y del PIB puede ser explicada desde dos puntos de vista, o incluso, la combinación de ambos. El primero, que exista un alto componente de trabajadores desanimados que abandonen la fuerza de trabajo tras una infructuosa búsqueda, situación que se conoce como desempleo oculto. El segundo, que los desempleados recurran al sector informal para resolver su necesidad de ingresos, con lo que pasan a ser clasificados como ocupados.

\section{Estado del arte}

El tiempo de búsqueda de empleo depende de muchos factores referidos tanto a la situación económica que genera el desempleo como a las características del propio trabajador. La literatura general analiza el efecto de algunas de estas variables. Así, el nivel educativo es visto como el factor determinante de la probabilidad de éxito a la hora de buscar empleo, aun cuando la direccionalidad de su efecto no es del todo clara. Así, mientras unos perciben que a mayor capacitación, mayor posibilidad de hallar empleo, otros consideran que este factor funciona en sentido opuesto, ya sea porque la educación eleva las aspiraciones salariales del trabajador, alargando su búsqueda, o porque la inadecuada estructura del aparato productivo favorece al trabajador menos capacitado, circunstancias que pueden variar incluso con el género (Bjorklund \& Eriksson, 1996; Bradley \& Nguyen, 2004; Bratberg \& Nilsen, 1998; Eckstein \& Wolpin, 1995; García, Carmona, \& Gómez, 2004; Kong, 2012; Kong \& Fan, 2011; Norman, 1984; Zhou, 2003). Otros estudios insisten en que los 
programas de ayuda a desempleados afectan positivamente la duración del desempleo, por cuanto los individuos solo tendrán incentivos para emplearse cuando agoten los beneficios del desempleo o cuando las ofertas laborales sean significativamente mejores que éstos, por lo que proponen regulaciones a las mismas ${ }^{2}$.

Las oportunidades de empleo no se encuentran igualmente distribuidas en el territorio nacional, producto de la situación económica particular de cada región, por lo que es obvio observar tiempos de búsqueda diferentes entre ellas (Ortega \& Martínez, 2005). La mayoría de los estudios que enfocan el problema de la búsqueda de empleo agrupan los mercados formal e informal, probablemente ante las dificultades para distinguir entre ellos. Otros, con acceso a mayor información, toman en cuenta las diferentes alternativas de decisión que encara el individuo, desagregando entre empleo formal, empleo informal, autoempleo y abandono de la fuerza laboral (Calderón, 2008), a lo que Abellán y Fernández (1997) agregan la emigración.

Además de los factores ya expuestos, la búsqueda de empleo puede verse afectada además por el género y edad del trabajador y la rama de actividad en la que intenta ocuparse. Un informe de la Oficina de Asesoría Económica y Financiera de la Asamblea Nacional de Venezuela (ANV) del 2003 estima la duración promedio del desempleo en Venezuela en 18,4 meses para las mujeres y 8,2 meses para los hombres. Según el informe, la búsqueda aumenta con la edad $(8,7$ meses para menores de 30 años y 24,9 meses para los mayores de 50 años) y el nivel educativo (16,8 meses para universitarios y 12,2 meses para los restantes niveles educativos). En algunos sectores, la búsqueda demora hasta 24 meses (electricidad, gas y agua) y se reduce a la mitad en otros (manufacturero). No se tiene información acerca de la metodología aplicada, por lo que los resultados no son necesariamente comparables con los obtenidos en este estudio.

\section{Metodología}

\section{Base de datos.}

La EHM es una investigación semestral que desde 1967 viene elaborando el INE, con el fin de recoger información acerca de la evolución del mercado laboral, la situación de vivienda y la estructura socioeconómica general del país. El panel construido para el periodo 2012-2013 consta de 30.270 individuos, 51,75\% de los cuales son mujeres. Para la primera cohorte, el $45,11 \%$ tiene educación básica o menos; 28,77 $\%$ es bachiller; $6,71 \%$ tiene título de técnico superior y el restante $19,41 \%$ es universitario. La edad promedio del grupo es de 34,12 años; la mayoría están casados o unidos (49,72\%), o solteros $(39,90 \%)$. Para el primer periodo, el $59,91 \%$ de los trabajadores está empleado; el $5,80 \%$ busca empleo y el resto forma parte de la población económicamente inactiva $(15,53$ $\%$ estudia, 14,29\% se dedica al hogar, 4,15 $\%$ incluye jubilados, rentistas, incapacitados u otros y el $0,33 \%$ no reporta ningún tipo de actividad, a pesar ser un grupo potencialmente productivo, cuya edad ronda los 26,5 años).

Este trabajo se enfoca en analizar el tránsito de ese porcentaje de individuos desempleados a lo largo de los cuatro periodos considerados, midiendo el tiempo que permanecen en esa condición y el nuevo estatus que adquieren al abandonarlo. Para ello, se parte del tiempo que el individuo lleva buscando para el primer semestre del 2012, ajustada por la información que reporta en las cohortes sucesivas, agregando paulatinamente el tiempo transcurrido entre cohortes, hasta que el individuo abandona la condición de desempleo.

\section{Metodología de análisis.}

El estudio parte del análisis descriptivo del tiempo de búsqueda de empleo, desagregado según algunas variables sociodemográficas, tales como género, nivel educativo, estado civil, región y ocupación, tanto para los desempleados 
en general como para los que buscan empleo por primera vez.

Para analizar la evolución de los individuos a través de los diferentes estados de la situación laboral se utiliza una cadena markoviana estacionaria finita, que tiene asociados un espacio de estados finito, una matriz estacionaria de probabilidades de transición $\mathrm{P}$ y un vector inicial de probabilidades. Sea $X_{t}$ el estado del proceso en el instante $t$, el cual representa la situación laboral en la que se encuentra el individuo en un momento dado. Los estados considerados, ocupado (Oc), desempleado (Ds), estudiante (Es), ama de casa (Ac), sin ninguna actividad $(\mathrm{Sa})$ y otros (Ot, que incluye jubilados, pensionados, discapacitados, rentistas) conforman el espacio de estados $\mathrm{E}=\{\mathrm{Oc}, \mathrm{Ds}, \mathrm{Es}, \mathrm{Ac}, \mathrm{Sa}, \mathrm{Ot}\}$. Es de particular interés para el estudio distinguir qué porción de los desocupados se mueve hacia la población económicamente inactiva y bajo qué figura lo hace, con miras a detectar situaciones de abandono; de allí la desagregación anterior.

La matriz de probabilidades de transición en $\mathrm{t}$ pasos $=\geq 0$ indica la probabilidad con la que se accede del estado $i$ al estado $j$ en $t$ periodos de tiempo (semestres). Se asume que el estado actual solo depende del estado inmediatamente anterior del sistema y que esta matriz es homogénea, regular e irreducible ${ }^{3}$ (Kemeny \& Snell, 1976).

\section{Resultados}

\section{Tiempo de búsqueda según factores sociodemogr“ ̈̈ cos.}

El panel señala que las tasas de desempleo se ubican en $8,82 \%$ y $7,66 \%$ en los dos semestres del 2012 y se mantienen similares en el primer y segundo semestres del 2013, con 8,22\% y 7,91\%, respectivamente. Estos valores son cónsonos con los reportados por el INE para el mismo periodo ${ }^{4}$. En promedio la búsqueda de empleo se prolonga durante 10,87 meses, tiempo que varía según el género y el nivel educativo. Los datos indican que el tiempo de búsqueda es significativamente mayor para las mujeres que para los hombres, lo que explica el que las tasas de desempleo sean mayores para el primer grupo $(8,9 \%$ versus 7,3 $\%$ ). Este fenómeno puede atribuirse a desiguales condiciones de acceso al mercado laboral o segmentación del mercado. Sin embargo, no es el propósito de este estudio ahondar sobre el tema. El tiempo de búsqueda aumenta con el nivel educativo. En efecto, el tiempo de búsqueda del universitario es significativamente superior al de trabajadores con nivel igual o inferior a bachiller, no así con respecto a los trabajadores con nivel de educación técnica (ver tabla 1). La teoría señala que este comportamiento se debe a que el salario de reserva aumenta con el nivel de estudios, por lo que el trabajador desecha aquellas ofertas laborales con remuneraciones inferiores a la esperada.

Tabla 1

Tiempo de búsqueda de empleo (meses) según género y nivel educativo 5

\begin{tabular}{|c|c|c|c|c|c|c|c|}
\hline & \multirow[b]{2}{*}{ General } & \multicolumn{2}{|c|}{ Género $^{\mathrm{a}}$} & \multicolumn{2}{|r|}{ Nivel } & \multicolumn{2}{|l|}{ educativo $^{b}$} \\
\hline & & $\begin{array}{c}\text { Hombres } \\
\text { (1) }\end{array}$ & $\begin{array}{c}\text { Mujeres } \\
\text { (0) }\end{array}$ & $\begin{array}{c}\text { Básica o } \\
\text { menos (1) }\end{array}$ & $\begin{array}{c}\text { Bachiller } \\
\text { (2) }\end{array}$ & $\begin{array}{c}\text { Técnico } \\
\text { Superior (3) }\end{array}$ & $\begin{array}{c}\text { Universitario } \\
\text { (4) }\end{array}$ \\
\hline Media & 10,87 & 10,46 & 11,34 & 10,64 & 10,77 & 11,32 & 11,37 \\
\hline D. E & 5,82 & 6,06 & 5,71 & 5,98 & 5,89 & 5,92 & 5,75 \\
\hline
\end{tabular}

Fuente: elaboración propia a partir de la Encuesta de Hogares por Muestreo [EHM], 2012-2013.

Nota: (a) $p(1,0)=0,00 ;$ (b) $p(F)=0,00 ; p(1,3)=0,06 ; p(1,4)=0,00 ; p(4,2)=0,01$. 
La duración del desempleo también es susceptible a la edad del trabajador, pero, a diferencia de lo que se espera, el grupo que más demora en abandonar la condición de desempleo es el de 21-35 años y el de menor tiempo es el de los mayores de 50 años, probablemente porque su edad les permite acceder a los beneficios de la jubilación. Este resultado es consistente con las mayores tasas de desempleo entre trabajadores en edades productivas, e inferiores en los mayores de 50 años, reportadas por el
INE. Se observan diferencias significativas en los tiempos de búsqueda entre todos los grupos, excepto entre 1 y 3 . La teoría también sostiene que los individuos casados o con algún tipo de compromiso familiar son más proclives a aceptar, y mantener, un empleo más prontamente que el trabajador soltero sin mayores responsabilidades (Borjas, 2013). Los datos no soportan esta hipótesis, al no encontrarse diferencias significativas entre los diferentes estados civiles considerados (ver tabla 2).

Tabla 2

Tiempo de búsqueda de empleo (meses) según grupo de edad y estado civil

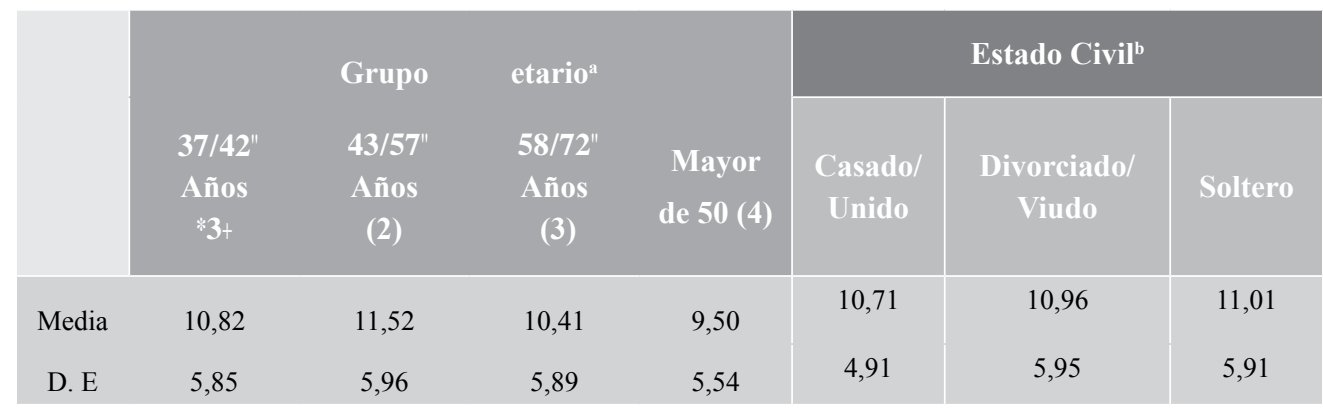

Fuente: Elaboración propia a partir de EHM, 2012-2013.

Nota: (a) $p(F)=0,00 ; p(1,2)=0,001 ; p(2,3)=p(1,4)=p(2,4)=p(3,4)=0 ;$ (b) $p(F)=0.08$

A nivel de regiones, solo destaca la región Centro-Norte con un tiempo de búsqueda significativamente inferior $(8,9$ meses), probablemente debido a la mayor actividad industrial, comercial y gubernamental que caracteriza esta región. Las demás regiones rondaron los $10 \mathrm{y}$ 11 meses, sin mayores diferencias que reportar. Ocupaciones relacionadas con el ejercicio de la ley tienen un promedio de espera de 9,18 meses en los hombres, inferior a los casi 12 meses que deben esperar las mujeres en la misma rama. Otras, como las no clasificadas, militares, diplomáticos, transporte, bienestar social y arte tienen un tiempo de espera inferior a los 10 meses en los hombres. Los profesionales de la salud de sexo masculino pueden demorar hasta 13,5 meses para abandonar la situación de desempleo. Es precisamente en este campo en el que las mujeres muestran los menores tiempos. No existen diferencias significativas en los tiempos de búsqueda entre ocupaciones dentro de cada grupo.

La duración del desempleo arroja luz sobre la naturaleza del problema y sus posibles causas. Una situación de búsqueda cercana o superior a un año es señal de desempleo de largo plazo, el cual está asociado al desempleo estructural. En el periodo considerado, el tiempo promedio que el individuo permanece en condición de desempleo se acerca demasiado a lo que pueden calificarse como desempleo de largo plazo. Más aún, el 36,5 \% de los desempleados muestran periodos de búsqueda superiores al año; de ellos, el $11,9 \%$ se mantienen en desempleo por más de año y medio. Lamentablemente, la información de la EHM no permite conocer qué porcentaje de estos desempleados tienen acceso a algún tipo de ayuda, como el seguro de paro forzoso ${ }^{6}$. 


\section{Tiempo de búsqueda para los debu- tantes.}

La búsqueda de empleo por primera vez impone el reto de lograr sortear el inconveniente de la falta de experiencia previa que la mayoría de los empleadores exige. Algunos países europeos ofrecen estímulos fiscales a las empresas que decidan ofrecer a los jóvenes debutantes la posibilidad de trabajar, política que la Organización para la Cooperación y el Desarrollo Económico (OCDE) espera sea aplicada por todos sus miembros; en América Latina, países como México y Uruguay han adelantado pasos en este sentido. La Organización Internacional del Trabajo -OIT (2005) advierte sobre los costos del desempleo prolongado entre los jóvenes, el cual puede incidir en los ingresos y en el nivel de competitividad del trabajador en el futuro.

Según reporta el INE, en Venezuela, la tasa de desempleo entre los jóvenes de 15 a 24 años de edad promedia $17 \%$ en los cuatro semestres del 2012 y 2013, mayor para las mujeres $(21,8 \%)$ que para los hombres $(14,3 \%)$, resultados que concuerdan con los obtenidos a partir de la muestra en estudio: 18,79\% para las mujeres y $15,92 \%$ de desempleo para los hombres, para un promedio general de 17,14 $\%$. Sin embargo, debe tenerse en cuenta que no solo los jóvenes en ese rango de edades buscan empleo por primera vez, por cuanto los datos señalan que la edad promedio de los debutantes ronda los 25 años, menor para los hombres (23,4 años) que para las mujeres (26,3 años), las cuales representan el $60 \%$ del total de ese grupo. El tiempo promedio de permanencia en situación de desempleo para los individuos que reportan estar buscando trabajo por primera vez se ubica en alrededor de 12 meses. Al igual que para el caso en general, estos tiempos son significativamente mayores para las mujeres y aumentan con el nivel educativo, con marcadas diferencias entre los dos niveles educativos más altos con respecto a los más bajos (ver tabla 3 ).

Tabla 3

Tiempo de búsqueda de empleo según género y nivel educativo para los que buscan trabajo por primera vez (meses)

\begin{tabular}{|c|c|c|c|c|c|c|c|}
\hline & \multirow[b]{2}{*}{ General } & \multicolumn{2}{|c|}{ Género $^{\mathrm{a}}$} & \multicolumn{2}{|r|}{ Nivel } & \multicolumn{2}{|l|}{ educativo $^{\mathrm{b}}$} \\
\hline & & $\begin{array}{l}\text { Hombres } \\
\text { (1) }\end{array}$ & $\begin{array}{c}\text { Mujeres } \\
\text { (0) }\end{array}$ & $\begin{array}{c}\text { Básica o } \\
\text { menos } \\
\text { (1) }\end{array}$ & $\begin{array}{c}\text { Bachiller } \\
\text { (2) }\end{array}$ & $\begin{array}{c}\text { Técnico } \\
\text { Superior (3) }\end{array}$ & $\begin{array}{c}\text { Universitario } \\
\text { (4) }\end{array}$ \\
\hline Media & 12,51 & 11,39 & 12,79 & 11,09 & 11,07 & 12,58 & 12,02 \\
\hline D. E & 4,97 & 4,85 & 4,89 & 4,79 & 4,8 & 4,61 & 4,75 \\
\hline
\end{tabular}

Fuente: elaboración propia a partir de EHM, 2012-2013.

Nota: (a) $p(F)=0,02 ;$ (b) $p(F)=0,03 ; p(1,3)=0,03 ; p(1,4)=0,02, p(2,3)=0,03 ; p(2,4)=0,02$.

Así visto, los datos parecen indicar que, si bien existen algunas diferencias en los tiempos de búsqueda de empleo entre grupos de edad, género, nivel educativo, e incluso ocupación y regiones, ningún grupo o sector sobresale por su facilidad para hallar empleo a corto plazo, lo que hace pensar que la prevalencia de desempleo de larga duración obedece definitivamente a un problema estructural, producto de la contracción general de la economía, la cual afecta a todos los sectores de la actividad económica y a todos los estratos socioeconómicos por igual. A mayor tiempo de búsqueda de empleo, mayor probabilidad de abandono de la misma (Borjas, 2013). De allí que resulte interesante conocer la transición de los desempleados por los diferentes estados de la condición laboral. 


\section{Transición a otros estados de situa- ción laboral.}

Como se dijo al inicio, la caída simultánea de la tasa de actividad económica y la tasa de desempleo puede explicarse, en parte, por el fenómeno del trabajador desanimado, el cual abandona la búsqueda cuando percibe que el esfuerzo es en vano. Del total de desempleados, el 65,11 $\%$ logra finalmente obtener un empleo, mientras que el $35 \%$ restante desiste del intento. Una buena parte de ellos retoma los estudios (12,75\% del total), en un intento por hacerse más competitivos; otros, casi $16 \%$, deciden permanecer en el hogar, mientras que el 5,3\% decide no hacer nada. Una muy pequeña proporción $(1,19 \%)$ se mantiene en la búsqueda o se mueve a otras condiciones como discapacitado, rentista o jubilado, ya sea prematuramente o a tiempo (ver tabla 4 ).
Esta migración de trabajadores potenciales de vuelta a la población económicamente inactiva, no solo constituye una pérdida de recurso productivo, sino que implica una subestimación de la tasa de desempleo, retardando o distorsionando el diseño y adopción de medidas que coadyuven a solventar el problema. La percepción de que no es fácil conseguir empleo se observa al preguntársele a los individuos las razones para no buscar empleo: más del $38 \%$ respondió que está cansado de buscar, cree que no hay trabajo o no hay trabajos apropiados; 10,3 $\%$ reconoce que no puede trabajar por problemas de organización y $11,3 \%$ está a la espera de un negocio o trabajo prometido. Los restantes, o bien no necesitan o no pueden trabajar, o no lo hacen por razones varias no especificadas. No se incluyen en el total a los que retoman los estudios.

Tabla 4

Destino de los trabajadores desempleados (\%)

\begin{tabular}{|c|ccccc|}
\hline $\begin{array}{c}\text { Número de } \\
\text { periodos }\end{array}$ & $\begin{array}{c}\text { Encuentran } \\
\text { empleo }\end{array}$ & $\begin{array}{c}\text { Retoman } \\
\text { estudios }\end{array}$ & $\begin{array}{c}\text { Permanecen en } \\
\text { el hogar }\end{array}$ & $\begin{array}{c}\text { No tiene ni } \\
\text { busca empleo }\end{array}$ & $\begin{array}{c}\text { Prosigue la } \\
\text { búsqueda / } \\
\text { Otro }\end{array}$ \\
\hline 1 & 79,76 & 94,19 & 89,88 & 86,87 & - \\
\hline 2 & 19,08 & 5,23 & 9,08 & 11,45 & - \\
\hline 3 & 1,16 & 0,58 & 1,04 & 1,68 & - \\
\hline Total & 65,11 & 12,75 & 15,65 & 5,30 & 1,19 \\
\hline
\end{tabular}

Fuente: elaboración propia a partir de EHM, 2012-2013.

Si bien la información disponible no permite indagar acerca de la posible adecuación de la calificación de los trabajadores para el trabajo que desempeñan, la revisión de algunas variables de la encuesta permite formarse una idea de la precariedad de los empleos, entendida ésta como poca estabilidad o corta duración. Del total de trabajadores que perdieron sus empleos por razones diferentes a jubilación, discapacidad o motivos personales, el 5,6 \% se retiró por inconformidad con los bajos salarios que recibía; $14,3 \%$ fue despedido por dificultades o cierre de la empresa; mientras que el 67,43\% tenía trabajos eventuales de corta duración. Ramoni (2012) alerta sobre la creciente incidencia del empleo a medio tiempo en Venezuela, con apenas $67 \%$ de los trabajadores empleados a tiempo completo en el año 2003. Según el panel en estudio, los individuos trabajan una media de 30,6 horas semanales, mientras que el 11,1 $\%$ de los desempleados que logran emplearse, trabajan 20 horas o menos a la semana.

La informalidad es otra consecuencia del desempleo estructural y los prolongados periodos de búsqueda de trabajo. Utilizando diferen- 
tes métodos, Ramoni, Orlandoni y Castillo (2010) estiman que el sector informal en Venezuela es responsable de entre 20 y $30 \%$ del PIB no petrolero para el 2007 y ocupa cerca de la mitad de los trabajadores. De acuerdo con el INE, en el periodo comprendido entre el primer semestre de 2012 y el segundo semestre del 2013, el $41,5 \%$ de los trabajadores estaban empleados en el sector informal. Para el primer semestre del 2012, la muestra señala que el 42,26 \% de los trabajadores se encuentran empleados en el sector informal, porcentaje que se mantiene muy similar para el segundo semestre del 2013 $(41,36 \%)$. De los desempleados que reportan haber conseguido empleo durante el periodo en estudio, el 47,86 \% lo hace en el sector informal; 38,82\% se emplea en la economía formal; y el $13,35 \%$ restante lo hace en actividades no clasificables. La combinación de un importante porcentaje de trabajadores abandonando la búsqueda de empleo y el desvío de la mitad de los que se emplean hacia el sector informal, pueden contribuir a explicar el porqué de la disminución en los niveles de desempleo, a la par que el PIB cae o se desacelera.

Sobre el sistema laboral en estudio, se define la variable aleatoria Situación Laboral, y se le asocia un conjunto de seis estados posibles (ocupado, desempleado, estudiante, hogar, otros, ninguno) a los que se les asigna cierta probabilidad de ocurrencia, definiéndose así una cadena de Markov en tiempo discreto. El sistema laboral parte de un estado inicial en el primer semestre del 2012, y los individuos se van moviendo entre estados, de manera recurrente, hasta alcanzar una distribución límite final o distribución de estado estacionario ${ }^{7}$ en el segundo semestre del 2013. Como los estados del sistema son de tipo recurrente, la comunicación entre ellos siempre es posible, generándose así las probabilidades que definen la matriz de transición asociada al sistema laboral en estudio.

Las matrices markovianas de transición representan la probabilidad de transición de un estado a otro de la situación laboral o, dicho de otro modo, la persistencia o fracción de individuos que permanecen en un mismo estado. La tabla 5 resume las probabilidades, en términos porcentuales, de transición de un estado a otro entre el primero y el último semestre del periodo en estudio; la estructura es muy similar a la observada periodo a periodo, condición requerida para poder estimar la probabilidad de persistencia a futuro.

Tabla 5

Matriz de transici ${ }^{1}$ n: periodo 2012:1 a 2013:2 (\%)

\begin{tabular}{|lcccccc}
\multicolumn{1}{r}{$\begin{array}{l}\text { Periodo } 4 \\
\text { Periodo } 1\end{array}$} & Ocupado & Desempleado & Estudiante & Hogar & Otros & Ninguno \\
\hline Ocupado & 73,46 & 4,66 & 8,49 & 10,01 & 3,23 & 0,15 \\
\hline Desempleado & 59,31 & 13,82 & 9,51 & 12,56 & 4,61 & 0,20 \\
\hline Estudiante & 42,82 & 5,37 & 36,29 & 9,83 & 5,32 & 0,37 \\
\hline Hogar & 44,97 & 4,77 & 8,55 & 36,94 & 4,38 & 0,39 \\
\hline Otros & 46,69 & 5,56 & 9,12 & 12,18 & 26,12 & 0,33 \\
\hline Ninguna & 46,78 & 11,70 & 11,70 & 22,81 & 5,26 & 1,75 \\
\hline Vector Estacionario & 63,71 & 5,31 & 11,90 & 14,04 & 4,82 & 0,22 \\
\hline
\end{tabular}

Fuente: elaboración propia a partir de EHM, 2012-2013.

En esta transición, destaca la creciente probabilidad tanto de permanecer desempleado como de que estudiantes se incorporen a la fuerza laboral, ya sea en calidad de ocupados o de desempleados. La creciente incorporación de estudiantes a la fuerza laboral puede estar 
asociada a la prevalencia de bajas remuneraciones, que obligan a todos los miembros del núcleo familiar a contribuir económicamente para subsistir. Según esta tabla, la probabilidad de permanecer en condición de desempleo entre el primer semestre del 2012 y último semestre del 2013 es de 13,82\%, mientras que la probabilidad de pasar del estado desempleado a empleado es $59,31 \%$. Asimismo, la proba- bilidad de abandonar la búsqueda y pasar a estado inactivo va de $12,56 \%$ a $0,20 \%$, dependiendo del sector de la población económicamente activa al que se mueva el individuo. La probabilidad de pasar a condición de desempleado estando ocupado inicialmente es muy baja, 4,66 \%. El esquema de esta transición general a partir de los resultados obtenidos se resume en la siguiente figura.

Figura 1

Esquema general de transición

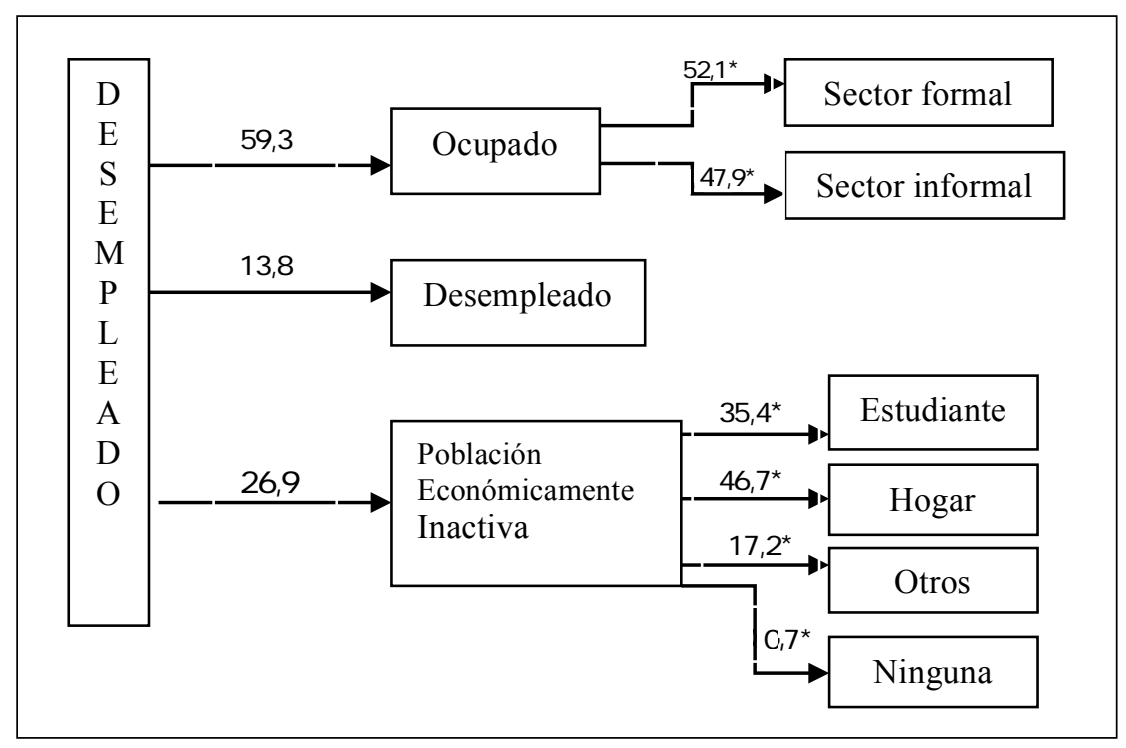

Fuente: elaboración propia a partir de EHM, 2012-2013.

Nota: (*) porcentajes respecto al subtotal de cada grupo.

El vector estacionario que se obtiene a partir de la matriz estocástica no debe entenderse como una predicción de lo que pueda suceder en el futuro. Solo es un indicador de la tendencia que sigue la distribución a partir del comportamiento de la variable analizada. Indica el porcentaje de individuos que pueden terminar en cada uno de los estados considerados, siempre que las condiciones socioeconómicas no sufran variaciones considerables. El proceso converge rápidamente hacia el vector de probabilidades estacionario resumido en la última fila de la tabla 5, el cual muestra el perfil de ocupación de largo plazo; representa la distribución límite o persistencia de largo plazo del sistema en estudio, y es independiente de su estado inicial.

Las únicas estimaciones de la distribución estacionaria que se tienen como referencia son las obtenidas por Márquez y Ruiz-Tagle (2004) para Venezuela en el periodo 1994-2002 utilizando otra metodología. En dicho estudio, la probabilidad de estar desempleado era $4,8 \%$, cercana al 5,31\% de nuestro vector; la probabilidad de permanecer empleado es mucho mayor: $84,9 \%$ versus $63,71 \%$; la probabilidad de persistencia de la población económicamente inactiva es apenas $10,3 \%$, muy por debajo del $30,98 \%{ }^{8}$ que arrojan nuestras estimaciones. Téngase presente que se trata de métodos y periodos diferentes. 


\section{Mecanismos de búsqueda de empleo.}

Trabajadores y empleadores acuden a diversos mecanismos para dar a conocer sus necesidades de empleo/empleados y lograr el mejor pareamiento trabajo-trabajador en el menor tiempo posible. Sin embargo, es conocido que existe un tradeoff o intercambio entre tiempo de búsqueda y calidad de empleo lo cual dependerá de terceros factores como, por ejemplo, el salario de reserva del trabajador, la propia apreciación del individuo en cuanto a lo que considera como empleo de calidad adecuado a sus expectativas y su capacidad de financiar sus gastos durante el tiempo que dure la misma. En esto juega un papel fundamental el seguro al desempleo, cuya normativa varía de un país a otro. Por ejemplo, mientras en Venezuela el seguro de desempleo ampara al trabajador por un periodo no mayor a cinco meses, en Estados Unidos el desempleado recibe hasta el $50 \%$ de su sueldo por un periodo de 33 semanas, ayuda que en algunos países de Europa puede extenderse por hasta tres años. La duración de esta ayuda incide directamente en el tiempo de búsqueda y, por ende, la tasa de desempleo (Brunner \& Calarelli, 2004).

El estudio de Márquez y Ruiz-Tagle (2004) ya citado resaltan la poca inclinación de los trabajadores a optar por agencias de trabajo, especialmente los que proceden del sector informal. Para la muestra en estudio, el análisis a las diligencias que llevan a cabo los desempleados para conseguir empleo revela que ese comportamiento no ha variado: más del $65 \%$ de los individuos recurren a métodos poco formales, esto es, contactos personales, mientras que menos del $4 \%$ hace uso de agencias de empleo. Nuestros datos no permiten conocer si los individuos emplean diferentes métodos de búsqueda simultáneamente.

Si bien no es el propósito de este estudio analizar el papel que estos mecanismos tienen sobre la probabilidad de éxito, resulta útil conocer el tiempo promedio de búsqueda para cada caso. Así, entre los individuos que lograron conseguir empleo, aquellos que contrataron los servicios de agencias especializadas lograron su objetivo en mucho menos tiempo $(8,72$ meses) que los que recurren a amistades y conocidos (10,5 meses). Contestar o colocar avisos y otras diligencias (créditos, permisos, etc.) reflejan tiempos de espera superiores al año. Se desconoce si el poco uso de agencias de empleo se debe a la escasa disponibilidad de las mismas a nivel nacional o si las condiciones de contratación de sus servicios resultan poco atractivas. Las agencias de empleo privadas pueden facilitar el conseguir empleos de mejor calidad en términos de remuneraciones, condiciones laborales y estabilidad, aunque no necesariamente reducir el tiempo de búsqueda, por lo que reforzar agencias de empleo podría ser un mecanismo recomendable para la reducción de la persistencia en la condición de desempleo y la mitigación del fenómeno del trabajador desanimado. Sin embargo, algunos estudios sugieren que esto no aplica a agencias de empleo públicas, las cuales demuestran ser más efectivas, pero menos eficientes (Addison \& Portugal, 2002; Gregg \& Wadsworth, 1996).

\section{Conclusiones y recomendaciones}

Al analizar el problema del desempleo, es importante evaluar las circunstancias que envuelven la transición del desempleado fuera de ese estado, incluyendo el tiempo que demora y la condición hacia la cual se mueve.

El panel construido con datos de la EHM de Venezuela para el periodo 2012-2013 permite concluir que el tiempo promedio de búsqueda de empleo es de aproximadamente once meses. Cuando se analiza la duración del desempleo según algunos criterios socioeconómicos, se observan ligeras diferencias, tales como búsquedas más prolongadas para las mujeres, para los niveles educativos más altos, o para ciertas regiones y ocupaciones. Este comportamiento puede atribuirse a elementos de desigual acceso al mercado de trabajo 
según género, relativamente más altos salarios de reserva o diferencias en la distribución de oportunidades por regiones y sectores. Sin embargo, el generalizado desempleo de largo plazo, sin ninguna característica en particular que permita atribuir ventaja alguna a un grupo por encima de otro, es propio de un problema estructural de la economía que afecta a todos los sectores por igual.

La prolongada búsqueda de empleo y la decreciente actividad económica reportada por las cifras oficiales, hacen obligante buscar explicaciones para la cada vez más baja tasa de desempleo reportada, que al cierre del periodo en estudio se ubica por debajo del $8 \%$. La propia EHM brinda las respuestas a esta interrogante. Poco más del $50 \%$ de los desempleados finaliza con éxito la búsqueda. El restante $35 \%$ se desincorpora de la fuerza laboral para retomar estudios y tratar de hacerse más competitivo, dedicarse a labores del hogar $u$ otras actividades. Casi el $48 \%$ de los que consiguen empleo, lo hacen en el sector informal, sin que se conozca la calidad de los empleos obtenidos por el $52 \%$ restante. No obstante, la muestra brinda información que permite sospechar una alta proporción de trabajadores con contratos de tiempo definido y empleos de medio tiempo. Así, la subestimación de las tasas de desempleo se explica tanto por el fenómeno de la informalidad como por el del abandono de la búsqueda, o trabajador desanimado. En efecto, casi la mitad de los individuos que no buscan trabajo por razones diferentes a que no lo necesitan, consideran que, o bien no hay empleos, o bien existen dificultades técnicas para iniciar una actividad laboral. Los mecanismos de búsqueda más utilizados tampoco son los idóneos, anteponiéndose el recurrir a contactos personales antes que utilizar métodos más formales.

Las matrices de transición apuntan a una creciente tasa de desempleo, de modo que, de mantenerse los problemas estructurales que condicionan el desempleo y su prolongada persistencia, la probabilidad (en términos porcentuales) a futuro de que un trabajador esté empleado es apenas inferior a $64 \%$, mientras que la probabilidad de que se ubique en la población económicamente inactiva supera el $30 \%$, más del doble del valor reportado por otro estudio para mediados de la década de los años 1990 y principios de los 2000. En todo caso, es obvio que la caída del desempleo observada no obedece a una paulatina solución a este problema.

De disponer paneles de datos más extensos, sería posible una visión más clara del problema en estudio. Igualmente, sería interesante tener información más actualizada, no disponible por retrasos propios del organismo emisor y dificultades para el acceso a la misma. Lamentablemente, el elevado sesgo de atrición no permite extender los paneles por más periodos sin una sustancial pérdida de información.

\section{Notas}

1 El rango de edades se justifica por la edad legal de trabajo y jubilación. El periodo considerado se justifica por tratarse de la información más reciente que pudo ser obtenida.

2 Para un estudio comparativo de los programas de asistencia al desempleado en países europeos, ver: Observatorio de Relaciones Laborales (2012).

3 Es homogénea por cuanto la probabilidad de transición es independiente del tiempo, es decir, $\mathrm{p}_{\mathrm{ij}}(\mathrm{t})=$ pij; es regular porque es posible acceder a cualquier estado del sistema en un número finito de períodos, independientemente del estado inicial del sistema; es irreductible y acíclica ya que todos sus estados se comunican un número indefinido de veces a intervalos no periódicos.

${ }^{4}$ Según el INE, las tasas de desempleo para los cuatro semestres fueron sucesivamente: $8,7 \%, 7,4 \%, 8,1 \%$ y $7,5 \%$. 
5 Para este y todos los demás gráficos, se indican solo las diferencias estadísticamente significativas. Los códigos entre paréntesis señalan los grupos.

6 Según la Ley de Régimen Prestacional de Empleo de 1985, modificada en la Asamblea Nacional 2005, este seguro cubre al trabajador por un periodo de hasta 5 meses con el equivalente al $60 \%$ de su sueldo mensual normal. No cubre a los que buscan empleo por primera vez, por cuanto se financia con los aportes previos del propio trabajador.

7 Se entiende por estado estacionario de una matriz de Markov homogénea aquel que se alcanza después de un determinado número de periodos y en el que las probabilidades de pertenecer a los diferentes estados se estabilizan en el tiempo (Kemeny \& Snell, 1976).

8 Este valor de 30,98\% resulta de sumar las probabilidades de los diferentes estados de inactividad económica, esto es $(11,90+$ $14,04+4,82+0,22)$.

\section{Referencias}

Abellán, C., \& Fernández, A. (1997). Relación entre la duración del desempleo y la probabilidad de emigrar (Documento de trabajo 141/97). España: Universidad de Oviedo.

Addison, J., \& Portugal, P. (2002). Job search methods and outcomes. Oxford Economic papers, 54, 505-533.

Asamblea Nacional de Venezuela -[ANV]. (2003). El desempleo en Venezuela: causas, efectos e implicaciones de política. Caracas: Oficina de Asesoría Económica y Financiera.

Bjorklund, A., \& Eriksson, T. (1996). Unemployment in the nordic countries. En E. Wadensjo (Ed), The nordic labour markets in the 1990's (pp. 96-116). Amsterdam: Elsevier.

Borjas, G. (2013). Labor Economics. New York: McGraw-Hill.

Bradley, S., \& Nguyen, A. (2004). The schoolto-work transition. En G. Johnes \& J. Johnes (Eds.), International Handbook of Education Economics (pp. 484-521). Cheltenham: Edward Elgar.

Bratberg, E., \& Nilsen, O. (1998). Transitions from School to Work: Search Time and Job Duration. IZA, 27, 1-29.

Brunner, L., \& Calarelli, S. (2004). Individual Unemployment Account. Independent Review, 8, 569-85.

Calderón, A. (2008). Unemployment dynamics in Mexico: Can micro-data shed light on the controversy of labor market segmentation in developing countries?. The Third Conference on Employment and Development. "WB" and "IZA", Morocco.

Di Tella, R., MacCulloch, R., \& Oswald, A. (2001). Preferences over Inflation and Unemployment: Evidence from Surveys of Happiness. American Economic Review, 91(1), 335-341.

Eckstein, Z., \& Wolpin, K. (1995). Duration to first job and the return to schooling: Estimates from a search-matching model. Review of Economic Studies, 62(2), 263-86.

García, J., Carmona, M., \& Gómez, J. (2004). La duración del tiempo de búsqueda del primer empleo. España: Universidad Católica San Antonio.

Gregg, P., \& Wadsworth, J. (1996). How effective are state employment agencies? Jobcenter use and job matching in Britain. 
Oxford Bulletin of Economics and Statistics, 58(3), 443-467.

Instituto Nacional de Estadística de Venezuela -[INE]. (2012-2013). Encuesta de Hogares por Muestreo -[EHM] 2012-2013. Venezuela: Autor.

Kemeny, J., \& Snell, J. (1976). Finite Markov Chains. New York: Springer-Verlag.

Kong, J. (2012). The analysis of graduate's job search by nonparametric survival approaches. Lecture notes in Information Technology, International Conference on Technology and Management Proceedings, 1- 21 .

Kong, J., \& Fan, J. (2011). Factors affecting job opportunities for university graduates in China. The evidence from university graduates in Beijing. Research in World Economy, 2(1), 24-36.

Márquez, G., \& Ruiz-Tagle, C. (2004). Search methods and outcomes in developing countries: The case of Venezuela (Working paper 519). Washington: InterAmerican Development Bank.

Norman, F. (1984). Factors Affecting Job Search Behavior and Employment Outcome for Youth. Princeton, N.J.: Educational Testing Service.

Observatorio de Relaciones Laborales. (2012). Paro de larga duración y protección por desempleo, ¿algo más que prestaciones?
Cauces. Cuaderno del Consejo Económico y Social, 21, 44-36.

Organización Internacional del Trabajo [OIT]. (2005). El empleo de los jóvenes: vías para acceder a un trabajo decente. Conferencia Internacional del Trabajo, 93.a reunión, Ginebra, Suiza.

Ortega, D., \& Martínez, I. (2005). Morfología del desempleo en Venezuela. En A. Freitez, J. Carbonel, A. Gruson, M. Phelan \& M. Villasmil (Eds.), Cambio demográfico $y$ desigualdad social en Venezuela al inicio del tercer milenio (pp. 359-377). Venezuela: AVEPO.

Ramoni, J. (2012). Descripción y análisis de los principales indicadores laborales. Venezuela: Banco Central de Venezuela; Consejo de Publicaciones ULA.

Ramoni, J., Orlandoni G., \& Castillo L. (2010). The size of the informal economy in Venezuela. El Norte- Finnish Journal of Latin American Studies, 5, 1-12.

República Bolivariana de Venezuela. Ley de Régimen Prestacional de Empleo de 2005. Gaceta Oficial No. 38.281. Asamblea Nacional de Venezuela. Recuperado de http://www.eumed.net/oe-vess/normas/ lrpe.htm

Zhou, J. (2003). A study on graduates' costs in job hunting. Economics of Education Research, 1(1), 12-20. 